\title{
System Message Based Predict Policy for Dynamic Power Management
}

\author{
Xi-Qiang Ma, Fang Yang, Ji-Shun Li \\ Henan Key Laboratory for Machinery Design and \\ Transmission System, Henan University of Science and \\ Technology \\ Luoyang, China \\ Email: mxq20149275@163.com
}

\author{
Yu-Jun Xue \\ Henan Key Laboratory for Machinery Design and \\ Transmission System, Henan University of Science and \\ Technology \\ School of Mechatronics Engineering, Henan University \\ of Science and Technology \\ Luoyang, China
}

\begin{abstract}
According to the traditional dynamic power management random policy can't also pointed out that moment of decision and state transition, dynamic power management improvement algorithm, SMBPP(System message based predict policy) is presented in this paper. Firstly, the existing dynamic power management policy algorithm which neglects the application characteristics of the workload is introduced. Secondly, based on the information of the system, the equipment utilization rate of the task is established, and the distribution is updated according to the actual interval time. Thirdly, the predict policy based on task equipment utilization is proposed, and the influence of the strategy parameters on the system sensitivity is analyzed.. Finally, the algorithm is used in wind power bearing state monitoring device. Experimental results show that with the performance constrain, the algorithm pointed out moment of decision and state transition, more stable and more effectively reduces the power consumption.
\end{abstract}

\section{Keywords-DPM; equipment utilization rate; predict policy.}

\section{INTRODUCTION}

With the development of semiconductor technology and the improvement of embedded device performance, power consumption on the system performance constraints becomes focus of embedded system research[1]. At this stage, low-power system design in general policy including dynamic power management (DPM) and dynamic voltage scaling (DVS), usually as a kind of DPM.

DPM policy in general can be divided into three categories: (a) Timeout policy, the basic idea is in the load after a timeout threshold will load the device into a low power state, including fixed timeout threshold method and adaptive timeout threshold policy[2]. (b) Predict policy, its essence is to forecast on the length of the idle time before making a decision, if the predicted value is large enough directly to PMC (power manageable component) to switch to the corresponding dormancy mode[3]. (c) Stochastic Markov policy, by establishing a Markov model to describe the stochastic behavior of the system device operation request and service, the DPM decision problem as a controlled Markov chain, thus more accurate choice decision time and equipment state transitions[4].

Timeout policy has the characteristics of simple principle and applied widely, but it is a kind of exploratory policy. Predict policy, once the threshold of inaccurate prediction, it may be counterproductive to increase power consumption. The study of stochastic Markov policy is very active in recent years. It can be divided into the following categories according to the establishment of the DPM decision model: based on modified Markov stochastic process level DPM model; based on continuous time Markov decision process DPM model; based on discrete time Markov decision process DPM model. DPM model of semi-Markov decision process based on time index, although the parameters of historical events are added, its essence is a kind of random timeout policy. Wu Qi theoretically proves the optimal strategies of DPM is deterministic Markov policy, and answer the timeout policy has very good performance and power consumption[5], but the DPM model ignores the interstate transition delay, the DPM control algorithm essentially belongs to the timeout policy, and on the assumption that the length of the idle time obeys the Pareto distribution, some limitations. All of the above policies ignore the application characteristics of the workload, and then cannot point out the decision time and the state of the transition. So this paper presents the SMBPP algorithm.

\section{EQUIPMENT UTILIZATION RATE}

The execution of a thread (the thread and process does not make a distinction in this paper) can according to equipment application program interface function by calling address division, the stack reflects the thread of the calling process, and can be used in stack depth and return address to distinguish the only device call path[6]. Stack depth is the deviation between the current stack pointer and the initial stack pointer, the return address is the current link register content, which can be stored in the context of the thread to get. So it can jointly predict task of equipment utilization rate according to application program interface function call stack depth and the return address. U(d,r) as equipment utilization rate (that is system history stack depth and return address correlation degree), is defined as follows:

$$
U(d, r)_{n}=a \bullet U(d, r)+(1-a) \bullet U(d, r)_{n-1}
$$

where a is discount factor,and it should be between 0.2 to $0.8[7]$.

Lookup table established by the U(d,r) elements stores the distribution of information, and it is required each thread device operation time interval distribution, the current task using time equipment accounted for the 
proportion of total working time of equipment, table lookup structure as shown in Tab 1, A lookup table example of five tasks (360rp, service, spoolsv, chrome and WINWORD) and six devices (TWI, TWI, EMAC, SPI, PWM and
TIMER) is given. Each operation request arrives according to return address stack depth and matching lookup table, and according to the actual mission equipment utilization rate update device lookup table.

TABLE I. EQUIPMENT UTILIZATION RATE WITH LOOKUP TABLE.

\begin{tabular}{|c|c|c|c|c|c|c|}
\hline & TWI & TWI & EMAC & SPI & PWM & TIMER \\
\hline $360 \mathrm{rp}$ & 0.02 & 0 & 0.64 & 0 & 0 & 0.21 \\
\hline service & 0 & 0.2 & 0.5 & 0 & 0 & 0.29 \\
\hline spoolsv & 0.16 & 0.02 & 0.23 & 0.04 & 0.1 & 0.4 \\
\hline chrome & 0 & 0.05 & 0.69 & 0 & 0.09 & 0.16 \\
\hline WINWORD & 0.41 & 0.02 & 0.05 & 0.2 & 0.03 & 0.44 \\
\hline
\end{tabular}

\section{SMBPP FOR DPM}

Varm[8] proposed the control theory method applied to dynamic voltage scheduling, and achieved good results. This paper puts forward SMBPP strategy referencing the basic idea: linear combination of the forecast value of the historical idle period, the actual value of the idle period and the idle period of the task equipment utilization rate is used to predict the next idle period length.

Proportion Integration Differentiation (PID) control is easy to use, strong adaptability and good robustness. By adjusting the PID parameters, the system energy saving effect tends to be optimal. General PID control of the expression is as follows:

$$
y(t)=K_{p} x+K_{I} \int_{0}^{t}(x-y) d t+K_{D} \frac{d x}{d t}
$$

According to the idea of general PID, we improve and transform it. First, the continuous time is converted to discrete time, that is, using the sum to replace the integral, using differential instead of differential, and the summation of infinite time for a limited period to the sum, or an average of just taking load variables past $m$ time value; then remove the $\mathrm{Y}$ item with the feedback feature at the right end of the equation, our objective is to predict the next idle period length, so after removing the feedback the system complexity is reduced; In the end, the xi is defined as the length of the $\mathrm{i}$ actual idle time, and the yi is defined as the length of the first $i$ estimate of the length of the idle time, and the PID equation is changed to:

$$
y_{n+1}=K_{P} \times x_{n}+K_{I} \times \sum_{n-m+1}^{n} \frac{x_{i}}{m}+K_{D} \times\left(x_{n}-x_{n-1}\right)
$$

The actual meaning of the proportional (3) part (the first item at the right end of the equation) is that the last time the actual idle time is the time of the next idle time, this ensures that the system can quickly track the changes in the load and if the load is suddenly changed, the system can respond quickly; the practical significance of the integral part (the second right end of the equation) is that the average time of history is regarded as the forecast time of the next free time, this smoothes the "burr" of the system load, so that the system tends to be stable; the practical significance of the difference in the right end of the equation (third) is that the rate of change of the last actual idle time is compensated by the time of the next idle time. KD represents the device utilization of the tasks mentioned in the last section, which is often encountered in the actual system.

The advantage of this strategy is that when the system load is stable, the output is stable; transient system load input fluctuations, will not lead to output fluctuations; the algorithm is convergent. In the process of strategy implementation, the key is the selection of parameters KP and KI. The sensitivity analysis of the system is shown in Figure 1. From Figure 1 we can see that when $\mathrm{KP}=40$ and $\mathrm{KI}=20$, the design space is relatively smooth.

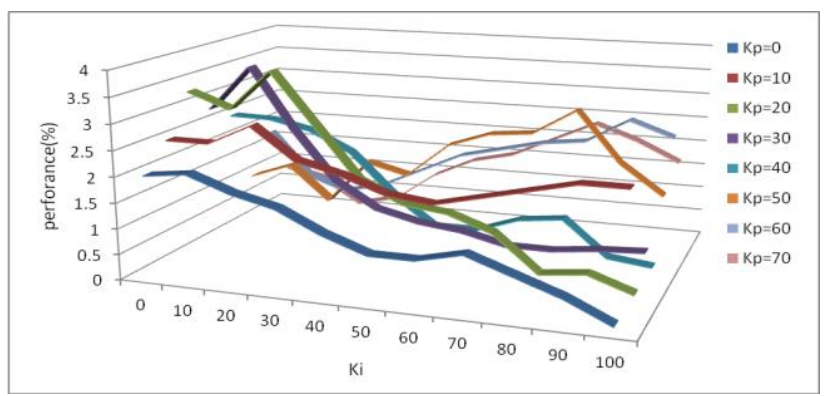

(a) Jitter of the system with variation 


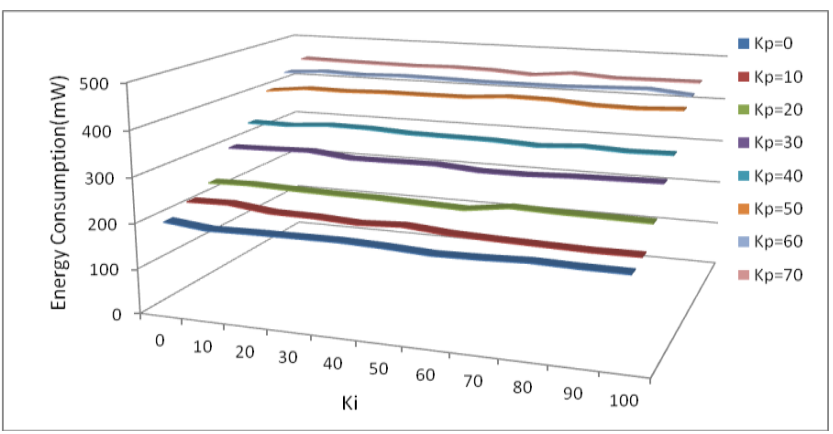

(b) System energy consumption with variation

Figure 1. Influence of the system to selection of SMBPP parameters

\section{EXPERIMENTAL RESULTS}

For strategic assessment of energy-saving effect and influence on the performance, this paper adopts the ADSPBF518 wind turbine bearings condition monitoring device based on as the experimental object, the algorithm with different timeout threshold timeout strategy and prediction strategy and random strategy are compared[9]. The ADSPBF518 processors are members of the Blackfin family of products with up to $400 \mathrm{MHz}$, provide various peripheral for networking, and run open ADSP uClinux real-time kernel to achieve the operating system flexibility, work at very low power levels. It is shown in Figure 2 and Figure 3.

ADSP-BF518 wind turbine bearings system is designed to demonstrate the ADSP-BF518F Blackfin processor capabilities and to be used in conjunction with the VisualDSP++ development environment. The VisualDSP++ development environment aids advanced application code development and debug.

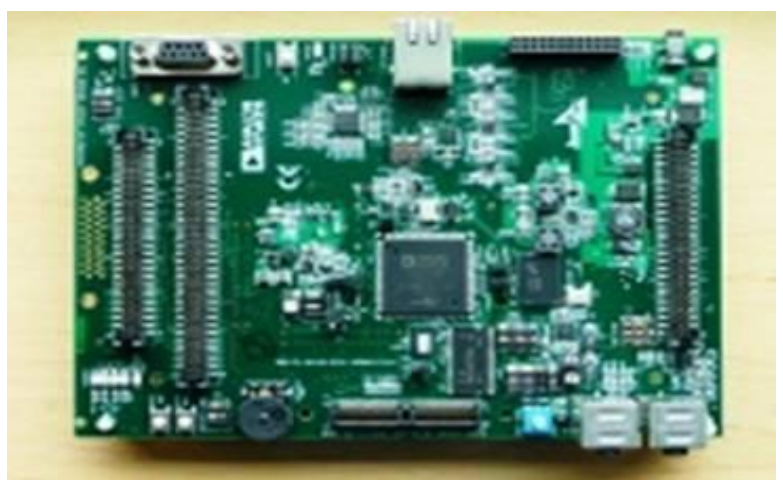

Figure 2. ADSP-BF518 system outline graph

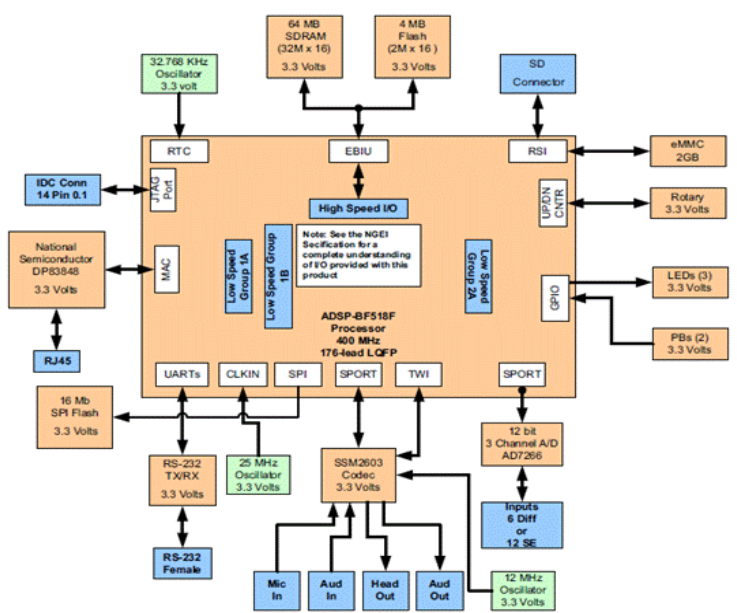

Figure 3. ADSP-BF518 system block diagram

Tab 2 shows the application device of PSM (power state machine) model. Each state are marked with the corresponding power, line marked on time and switching power state transitions are required.

TABLE II. PSM MODULE OF THE ADSP-BF518 SYSTEM

\begin{tabular}{|c|c|c|c|}
\hline & state 1 & state 2 & state 3 \\
\hline state 1 & power $=52 \mathrm{~mW}$ & power $=20 \mathrm{~mW}$ & power $=130 \mathrm{~mW}$ \\
& $\mathrm{~T}=0 \mathrm{us}$ & $\mathrm{T}=30 \mathrm{us}$ & $\mathrm{T}=90 \mathrm{us}$ \\
\hline state 2 & power $=3 \mathrm{~mW}$ & power $=90 \mathrm{~mW}$ & power $=62 \mathrm{~mW}$ \\
& $\mathrm{~T}=4 \mathrm{us}$ & $\mathrm{T}=0 \mathrm{us}$ & $\mathrm{T}=40 \mathrm{us}$ \\
\hline \multirow{2}{*}{ state 3 } & power=11mW & power $=8 \mathrm{~mW}$ & power $=280 \mathrm{~mW}$ \\
& $\mathrm{~T}=15 \mathrm{us}$ & $\mathrm{T}=10 \mathrm{us}$ & $\mathrm{T}=0$ us \\
\hline
\end{tabular}

In the experimental environment, to verify the effect produced a column six different tasks. Each task column is applied to the following power management policy (Tbe is defined as the equilibrium time[12]):
(1) CTMDP[4]: continuous time stochastic Markov policy;

(2) Timeout: fixed time timeout policy with timeout threshold $=$ Tbe; 
(3) Adaptive[10]: adaptive policy with timeout threshold $=$ Tbe, if system achieves the timeout threshold the state 2 is transformed;

(4) DTMDP[11]: discrete time stochastic Markov policy;

(5) This paper: system message based predict policy.

Using the competitive rate $\zeta$ as the evaluation index of power consumption, the delay rate $\sigma$ as the performance index, the hit rate $\eta$ as the prediction efficiency index of the strategy, are defined as follows:

$$
\varsigma=\left(E_{n o}-E_{A}\right) /\left(E_{n o}-E_{o p t}\right)
$$

Where EA is the energy consumption of the current strategy, Eno is energy consumption without any strategy, and Eopt is the energy consumption under the optimal offline strategy.

$$
\delta=\left(T_{A}-T_{n o}\right) / T_{n o}
$$

Where TA is the total time under the current policy, Tno is the total time without any strategy.

$$
\eta=\text { hit the correct number } / \text { Predicted total number }
$$

Competitive ratio $\zeta$ represents the power efficiency of the current policy with respect to the optimal policy. The competitive rates of each strategy are shown in Figure 4 (a). It can be seen from the figure, this paper's power efficiency of the strategy is significant, higher from the adaptive strategy $2 \%$, and the average value reached 0.47 . CTMDP has the lowest power consumption efficiency, this is because the CTMDP strategy is the user to obey the exponential distribution based on the assumption, resulting in idle time and lack of equipment long closing times increasing, and this strategy also needs periodic model, consume a lot of energy.

The delay rate and hit rate of each strategy are shown in Figure 4 (b) and Figure 4 (c) respectively. Adaptive policy has a lower rate of delay and higher hit rate, the competition rate of Predict policy has greater volatility. Timeout policy only accurately selected timeout threshold, and the energy saving effect is close to optimal, otherwise the effect is not ideal, make decisions on the same system, its estimated value is different, so the exact timeout threshold is very difficult to get. Overall, in addition to the strategy in this paper, the delay rate is less than 0.11 , and other strategies are more than 0.25 .

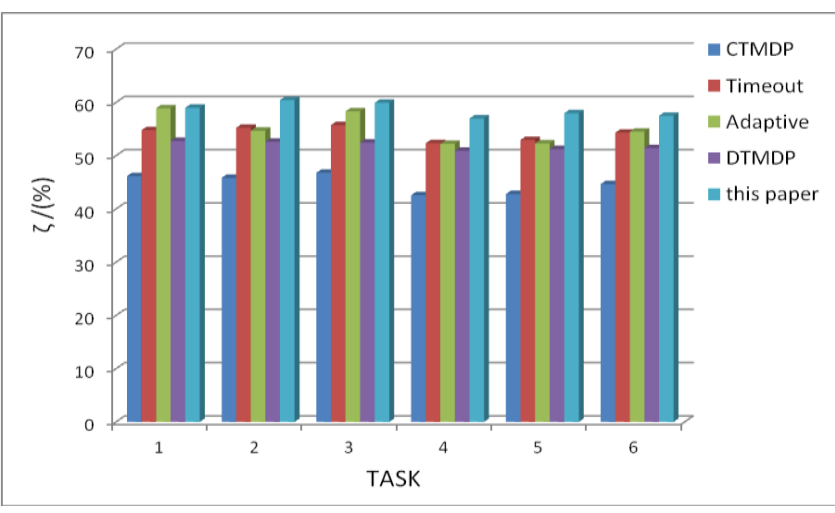

(a)

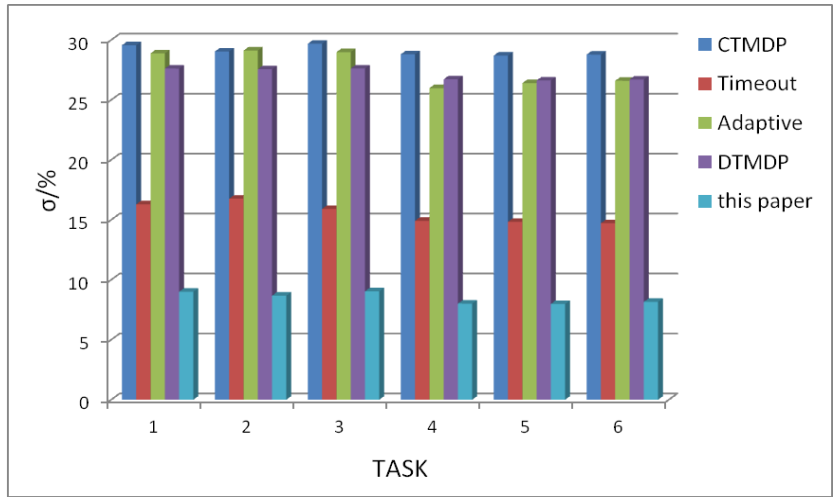

(b)

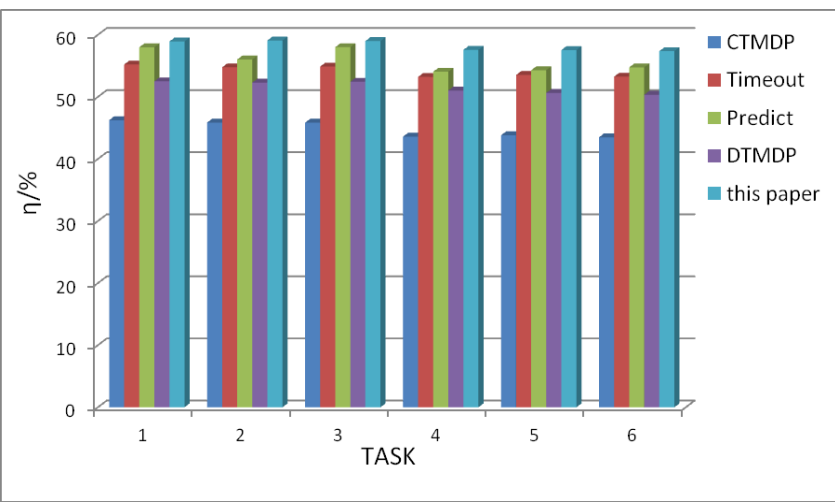

(c)

Figure.4. Comparison of competitive ratios, delay ratios and hit ratios for all policies

\section{CONCLUSIONS}

For embedded system of multi task environment nonstationary characteristics, this paper put forward the SMBPP algorithm. Through the stack depth and the return address to joint distinguish device call path, the decision making based on random semi Markov model of task level. Stochastic optimal algorithm is given in this paper from the length of the idle time distribution limits and can be applied 
to general distribution and gives the determining method of decision time and decision strategy chosen, algorithm to calculate the amount of small, between the energy efficiency and system performance find a compromise entry point. Needs to be pointed out is that the final part strategy properly simplify the system model, the result is only a reference; secondly, Markov model based on information system is to estimate the probability of a complex and stochastic process, for the same system its estimated value is possibly different, but are obtained through the method of the final strategy tends to be optimal. The experimental results indicate that this delay rate is less than 0.11 , and the competition rate can reach 0.47 . It is conducive to the application in embedded system.

\section{ACKNOWLEDGEMENTS}

This work is supported by the National Key Basic Research Program (973) of China (2014CB049401), the Project of Basic and Advanced Technology Research of Henan Province of China (152300410081), the Project of Basic and Advanced Technology Research of Henan Province of China (162300410034), the Key Science and Research Program in University of Henan Province (15A460018), and the Program for Innovative Research Team (in Science and Technology) in University of Henan Province (15IRTSTHN008).

\section{REFERENCES}

[1] ZHOU WH, WU J, ZHONG WF. Electricity Consumption Scheduling with Consumers' Comfort and Preference in Smart Grid[J]. Chinese Journal of Electronics, 25(6):1151-1158(2016).

[2] Cao Z, You Z. The influencing factor of threshold selection in dynamic threshold of timeout policies[J]. Journal of Harbin Institute of Technology, 45(6):119-123(2013).
[3] CHENG CL, WANG Y, ZHANG DY. Strategy of servers awakening based on dynamic threshold in cloud computing[J] Systems Engineering and Electronics, 37(6): 1437-1445(2015).

[4] Jiang Q, Xi HS, Yin BQ. Stochastic switching model and policy optimization for dynamic power management[J]. JOURNAL OF COMPUTER-AIDED DESIGN \& COMPUTER GRAPHICS, 18(5): 680-686(2006).

[5] Wu Q, Xiong GZ. Adaptive dynamic power management for nonstationary self similar requests[J]. Journal of Software, 16( 8): 1499 1505(2005).

[6] Qi LN, Zhang Z, Huang SM. Dynamic power management for I/O devices under multi-task environment[J]. Engineering Science, 10(2): 60-65(2008)

[7] Hwang CH, Wu ACH. A Predictive System Shutdown Method for Energy Saving of Event-Driven Computation[J]. ACM TRANSACTIONS ON DESIGN AUTOMATION OF ELECTRONIC SYSTEMS, 5(2): 226-241(2000).

[8] Varma, A., Ganesh, B., Sen, M., Choudhury, S. R., Srinivasan, L., \& Jacob, B. (2003). A control-theoretic approach to dynamic voltage scheduling. International Conference on Compilers, Architectures and Synthesis for Embedded Systems, CASES 2003, San Jose, California, Usa, October 30 - November (pp.255-266).

[9] Wu, C., Mohsenianrad, H., \& Huang, J. (2012). Wind power integration via aggregator-consumer coordination: A game theoretic approach. Innovative Smart Grid Technologies (pp.1-6). IEEE.

[10] Lu YH, De Micheli G. Comparing system-level power management policies[J]. IEEE DESIGN \& TEST OF COMPUTERS, 18(2): 10-19(2001).

[11] Benini L, Bogliolo A, Paleologo GA, et al. Policy optimization for dynamic power management[J]. IEEE TRANSACTIONS ON COMPUTER-AIDED DESIGN OF INTEGRATED CIRCUITS AND SYSTEMS, 18(6): 813-833(1999).

[12] Wai-Kong Lee, Sze-Wei Lee, Wee-Ong Siew. Hybrid model for dynamic power management[J]. IEEE Transactions on Consumer Electronics, 55(2): 656-664(2009). 